\title{
Multi-Objective Capacitated Solid Transportation Problem with Uncertain Variables
}

\author{
Vandana Y. Kakran \\ Department of Mathematics and Humanities, \\ S. V. National Institute of Technology, Surat-395007, Gujarat, India. \\ Corresponding author: vandana.kakran98@gmail.com \\ Jayesh M. Dhodiya \\ Department of Mathematics and Humanities, \\ S. V. National Institute of Technology, Surat-395007, Gujarat, India. \\ E-mail: jdhodiya2002@yahoo.com
}

(Received on May 4, 2021; Accepted on September 22, 2021)

\begin{abstract}
This paper investigates a multi-objective capacitated solid transportation problem (MOCSTP) in an uncertain environment, where all the parameters are taken as zigzag uncertain variables. To deal with the uncertain MOCSTP model, the expected value model (EVM) and optimistic value model (OVM) are developed with the help of two different ranking criteria of uncertainty theory. Using the key fundamentals of uncertainty, these two models are transformed into their relevant deterministic forms which are further converted into a single-objective model using two solution approaches: minimizing distance method and fuzzy programming technique with linear membership function. Thereafter, the Lingo 18.0 optimization tool is used to solve the single-objective problem of both models to achieve the Pareto-optimal solution. Finally, numerical results are presented to demonstrate the application and algorithm of the models. To investigate the variation in the objective function, the sensitivity of the objective functions in the OVM model is also examined with respect to the confidence levels.
\end{abstract}

Keywords- Capacitated solid transportation problem, Uncertain variable, Optimistic value model, Fuzzy programming technique.

\section{Introduction}

Transportation is an integral component of the growing local and global economy of the world these days. The traditional transportation problem (TP) is a well-known optimization problem that was initially introduced by Hitchcock (1941) to deal with the transportation system. He addressed two main constraints in the TP, primarily source constraints and demand constraints with an aim to determine the minimum transportation cost for supplying goods from various source centres to destination centres. However, in the physical world, we frequently encounter numerous situations where, in addition to the source-destination constraints, other constraints associated with product type or modes of transportation (conveyances) are also present. Such TP is known as the solid transportation problem (STP), and it was first presented by Schell (1955). Sometimes, due to various factors like road safety, storage limitations or budget issues, decision-makers may specify the total capacity of each route. As a result of these circumstances, Wagner (1959) proposed the concept of capacitated TP. When STP is studied along with capacitated TP, it is known as capacitated STP (CSTP). As the basic form of TP consists of a single-objective only, when multiple objectives are introduced simultaneously in the CSTP, then it is termed as multi-objective CSTP (MOCSTP). Zimmermann (1978) introduced a fuzzy programming approach for solving TPs with multiple objective functions. Lohgaonkar and Bajaj (2010) used this technique to solve multi- 
objective CTP (MOCTP). In 2014, Gupta and Bari (2014) applied the same technique for solving MOCTP with mixed constraints.

The capacitated transportation problem has been a major area of research among the researchers. Panda and Das (2014) represented a two-vehicle transportation cost varying model to solve CTP in which the cost varies due to capacity of vehicles as well as amount of transport quantity. Acharya (2016) considered a generalized solid CTP and developed a solution procedure to find the optimal solution. Ahmadi (2018) obtained the solution for CTP with the modification of three existing methods: North West corner method, least cost method, and Vogel's approximation method. Recently, Sharma and Arora (2021) explored a class of bi-objective capacitated TP with bounds over supply capacity of sources and demand requirements at destinations, and its solution is obtained using an iterative algorithm.

The research work cited so far assumes that the parameters involved in the TPs are precisely known. However, in some cases, it may not be possible to define parameters with precise or accurate values. Several factors contribute to this inaccuracy (such as insufficient or inexact information, weather conditions or market fluctuations). Therefore, many researchers developed different theories like fuzzy set theory (Zadeh, 1996), probability theory (Kolmogorov and Bharuch-Reid, 2018), and interval theory (Moore and Yang, 1996) so as to represent imprecise parameters for TPs. Hassin and Zemel (1988) studied the probabilistic analysis of CTP under the random uncertain environment. Many researchers like Bhargava et al. (2014); Giri et al. (2014); Ebrahimnejad (2015) have considered the TP with multiple objectives in the fuzzy domain. Sadia et al. (2016) found a compromise solution for a MOCTP with fractional objectives and mixed constraints using lexicographic goal programming (GP) and fuzzy programming. Gupta et al. (2018) obtained the solution of the multi-choice MOCTP with uncertain demand and supply using the GP model. They have also presented a paper on extended MOCTP with mixed constraints under the fuzzy environment and obtained the compromise solution by developing a new weighted GP model (Gupta et al., 2020).

As per Liu (2007), we utilize probability theory when we have a sufficient amount of available data based on historical information to predict the probability distribution. But under certain situations, there are no points of reference accessible to predict this distribution, and at that point, the degree of belief is being estimated by the researchers for the occurrence of each event. Liu (2007) presented uncertainty theory which dealt with the belief degree of humans, and later it has been enhanced by Liu (2010). In 2009, he introduced the theory of uncertain programming (Liu and Liu, 2009). Cui and Sheng (2013) developed a model for the STP in the uncertain environment and obtained its solution using the simplex method after transforming the uncertain model into its deterministic form using uncertainty theory. Mou et al. (2013) studied the TP by considering the truck times and unit costs as uncertain variables, and developed a heuristic algorithm based on a stepwise optimization strategy to obtain its solution. Gao and Kar (2017) considered the STP with blending of product in the uncertain environment. Chen et al. (2019) proposed an entropy-based STP in the uncertain environment, where the entropy function was employed as a second objective function so as to ensure the uniform delivery of goods between sources and destinations. Zhao and Pan (2020) focussed on the transportation planning problem by taking the transfer costs in an uncertain environment. Kakran et al. (2021) obtained the solution of uncertain TP with multiobjectives utilizing fuzzy programming approach and weighted sum approach. 
A major key problem in uncertain programming models is to rank the uncertain variables. As a result, four criteria were introduced by Liu (2010) to rank the uncertain variables in the uncertain model. These ranking criteria are listed as: expected value criterion (EVC), optimistic value criterion (OVC), pessimistic value criterion (PVC) and chance-criterion (CC). As far as we are concerned, the MOCSTP has not been studied with the uncertain theory given by Liu (2007). So, this study is instigated to deal with the real-life MOCSTPs, where one wants to optimize multiple objectives simultaneously while transporting products from available sources to destinations using different conveyances with capacitated constraints under the uncertain environment (rather than fuzzy or random environment). To deal with the uncertain model of MOCSTP, we have developed the EVM and OVM models using the expected and optimistic value criterion to rank the uncertain variables. The uncertain MOCSTP model is transformed into its deterministic model by formulating the OVM model which differs from the existing work in the literature. The deterministic form of the uncertain model has also been obtained by formulating the expected value model, which is a renowned model among the researchers to handle the uncertain models. The results of EVM and OVM models are acquired by utilizing the two classical approaches: minimizing distance method and fuzzy programming technique. These two solution approaches find a wide number of applications in the uncertain transportation problems with multiple objectives because of their simple and efficient use.

The further sections of this paper are organised as follows. Section 2 highlights some important notions on uncertainty theory that are necessary for the study of this paper. Section 3 states the mathematical description of MOCSTP followed by section 4 that introduces the uncertain model for the MOCSTP, and its respective EVM and OVM uncertain models are constructed. Section 5 provides the relevant deterministic models for EVM and OVM uncertain models using uncertainty theory. The next section 6 gives the two solution methodologies used for solving the deterministic models of MOCSTP. Section 7 provides a numerical illustration to depict the application of the models. In section 8, sensitivity of the objective functions involved in the OVM model is analysed w.r.t. the confidence levels.

\section{Introduction to Uncertainty Theory}

This section defines and introduces several key concepts in the field of uncertainty theory.

Definition 2.1 Liu (2010): A function $M: \mathfrak{I} \rightarrow[0,1]$ (where $\mathfrak{I}$ is a $\sigma$-algebra over any non-empty set $\Gamma$ ) that meets the stated axioms, is known as an uncertain measure.

Axiom $1 M\{\Gamma\}=1$.

Axiom $2 M\{\Lambda\}+M\left\{\Lambda^{c}\right\}=1$, for event $\Lambda \in \mathfrak{J}$.

Axiom $3 M\left\{\bigcup_{j=1}^{\infty} \Lambda_{j}\right\} \leq \sum_{j=1}^{\infty} M\left\{\Lambda_{j}\right\}$ for any countable sequence of events $\left\{\Lambda_{j}\right\}$.

Here, the space denoted by the triplet $(\Gamma, \mathfrak{I}, M)$ is known as an uncertainty space.

Definition 2.2 Liu (2010): A measurable function $\eta$ from uncertainty space $(\Gamma, \mathfrak{J}, M)$ to $\mathfrak{R}$ such that $\{\eta \in B\}$ is an event for any Borel set $B$ of real numbers, is known as ancertain variable. 
Definition 2.3 Liu (2010): The uncertainty distribution function $\Phi: \Re \rightarrow[0,1]$ for an uncertain variable $\eta$ is defined by $\Phi(y)=M\{\eta \leq y\}, y \in \mathfrak{R}$.

Definition 2.4 Liu (2010): An uncertain variable $\eta$ with $\Phi(y)$ defined as

$$
\Phi(y)= \begin{cases}0, & \text { if } \leq p, \\ \frac{y-p}{2(q-p)}, & \text { if } p \leq y \leq q, \\ \frac{y+r-2 q}{2(r-q)}, & \text { if } q \leq y \leq r, \\ 1, & \text { if } y \geq r .\end{cases}
$$

is called zigzag uncertain variable denoted by $Z(p, q, r), p, q, r \in \mathfrak{R}$ and $p<q<r$.

Definition 2.5 Liu (2010): The inverse uncertainty distribution function denoted by $\Phi^{-1}$ of $Z(p, q, r)$ is given by

$\Phi^{-1}(\beta)= \begin{cases}(1-2 \beta) p+2 \beta q, & \text { if } \beta<0.5, \\ (2-2 \beta) q+(2 \beta-1) r, & \text { if } \beta \geq 0.5 .\end{cases}$

Definition 2.6 Liu (2010): An uncertain variable $\eta$ has the expected value given by $E[\eta]=\int_{0}^{1} \Phi^{-1}(\beta) d \beta$, if it exists. The expected value of $Z(p, q, r)$ is given by $E[\eta]=\frac{p+2 q+r}{4}$.

Definition 2.7 Liu (2010): For any two independent uncertain variables $\eta$ and $\xi$, $E[p \eta+q \xi]=p E[\eta]+q E[\xi], p, q \in \mathfrak{R}$.

Definition 2.8 Liu (2010): The $\beta$-optimistic and $\beta$-pessimistic values for $Z(p, q, r)$ are defined by

$\eta_{\text {sup }}(\beta)= \begin{cases}(2 \beta q+(1-2 \beta) r, & \text { if } \beta<0.5, \\ (2 \beta-1) p+(2-2 \beta) q, & \text { if } \beta \geq 0.5 .\end{cases}$

$\eta_{\text {inf }}(\beta)= \begin{cases}(1-2 \beta) p+2 \beta q, & \text { if } \beta<0.5, \\ (2-2 \beta) q+(2 \beta-1) r, & \text { if } \beta \geq 0.5 .\end{cases}$

Theorem 2.9 Liu (2010): For any uncertain variable $\eta$ and $\beta \in(0,1]$, we have:

(a) $\eta_{\text {sup }}(\beta)$ is a left-continuous and decreasing function of $\beta$.

(b) $\eta_{\text {inf }}(\beta)$ is a left-continuous and increasing function of $\beta$. 
The critical problem with uncertain variables is that, they do not obey any justified order in the uncertain environment. So, for any two uncertain variables $\eta$ and $\xi$, Liu (2009) gave four ranking criteria which are EVC, OVC, PVC and CC.

EVC states that $\eta<\xi$ iff $E[\eta]<E[\xi]$.

OVC states that $\eta<\xi$ iff $\eta_{\text {sup }}(\beta)<\xi_{\text {sup }}(\beta)$ for some $\beta \in(0,1]$.

PVC states that $\eta<\xi$ iff $\eta_{\text {inf }}(\beta)<\xi_{\text {inf }}(\beta)$ for some $\beta \in(0,1]$.

CC states that $\eta<\xi$ iff $M\{\eta \geq \bar{r}\}<M\{\xi \geq \bar{r}\}$ for some predefined level $\bar{r}$.

\section{Problem Description}

This section describes the MOCSTP with an assumption of $m$ sources, $n$ destinations, and $K$ conveyances. MOCSTP concerns with developing an ideal transportation plan with the objective of optimizing all the multiple objective functions, which can be either transportation cost, transportation time or damage cost etc. The MOCSTP model can be mathematically written as shown below:

\section{Model 3.1}

$\min Z^{t}=\sum_{\forall i} \sum_{\forall j} \sum_{\forall k}\left(c_{i j k}^{t} x_{i j k}\right), \forall t$

Subject to the constraints:

$$
\begin{aligned}
& \sum_{\forall j} \sum_{\forall k} x_{i j k} \leq a_{i}, \forall i, \\
& \sum_{\forall i} \sum_{\forall k} x_{i j k} \geq b_{j}, \forall j, \\
& \sum_{\forall i} \sum_{\forall j} x_{i j k} \leq e_{k}, \forall k, \\
& \mathrm{O} \leq x_{i j k} \leq l_{i j k} ;
\end{aligned}
$$

Here, $m$ represents the total sum of available sources, $n$ represents the total sum of available destinations, $K$ represents the total sum of available modes of transportation, $a_{i}$ represents the capacity of source $i, b_{j}$ represents the total requirements at destination $j$, and $e_{k}$ is the maximum capacity of the conveyance $k$ during transportation. In addition, $c_{i j k}^{t}$ represents the transportation cost per unit of item from source-destination pair $(i, j)$ using conveyance $k$ for objective $t, x_{i j k}$ represents the number of transported items from source-destination pair $(i, j)$ with conveyance $k$, and $l_{i j k}$ represents the total restriction on $x_{i j k}$ for transportation from source-destination pair $(i, j)$ using conveyance $k$. In this paper, we have used the notations, $\forall t$ for $t=1,2, \ldots S, \forall i$ for $i=1,2, \ldots m, \forall j$ for $j=1,2, \ldots n$, and $\forall k$ for $k=1,2, \ldots K$. 
The $S$ objective functions in MOCSTP model aim to minimize the total transportation cost or damage cost etc. for delivering items to all the $n$ destinations from available $m$ sources using $K$ conveyances. The supply constraint given by equation (1) denotes that the number of transported items from the source $i$ to total $n$ destinations using $K$ conveyances cannot exceed the total supply capacity of the source $i$. The demand constraint given by equation (2) states that the number of transported items from different suppliers using $K$ conveyances should fulfil the demand requirements at destination $j$. The conveyance constraint shown in equation (3) states that the number of transported items should not exceed the capacity of the conveyance $k$. Lastly, we have the capacitated constraints given by equation (4) which gives the total restriction on $x_{i j k}$ for transportation from source-destination pair $(i, j)$ using conveyance $k$.

The above model (3.1) assumes all the variables $c_{i j k}^{t}$ and $a_{i}, b_{j}, e_{k}$ as constants. But in practical situations, we are not able to define these variables accurately due to lack of information as the transportation plan is supposed to be made in advance. If the previously used information regarding the plan is available, the variables can be treated as random variables but if we are not provided with the previous information then treating these variables as the random variables will not lead us to the appropriate results. Thus, in such cases, when we have lack of information about the historical data, we take into consideration the concepts of uncertainty theory given by Liu (2007). So, the variables $c_{i j k}^{t}$ and $a_{i}, b_{j}, e_{k}$ in the MOCSTP are replaced by $\xi_{i j k}^{t}$ and $\tilde{a}_{i}, \tilde{b}_{j}, \tilde{e}_{k}$ respectively in the uncertain environment which are called uncertain variables. Then the MOCSTP becomes uncertain MOCSTP, denoted by UMOCSTP.

\section{Uncertain Mathematical Model}

Replacing the uncertain variables $\xi_{i j k}^{t}$ and $\tilde{a}_{i}, \tilde{b}_{j}, \tilde{e}_{k}$ in the model (3.1), we get the following model:

\section{Model 4.1}

$\min Z^{t}(x ; \xi)=\sum_{\forall i} \sum_{\forall j} \sum_{\forall k}\left(\xi_{i j k}^{t} x_{i j k}\right), \forall t$;

Subject to the constraints:

$$
\begin{aligned}
& \sum_{\forall j} \sum_{\forall k} x_{i j k} \leq \tilde{a}_{i}, \forall i, \\
& \sum_{\forall i} \sum_{\forall k} x_{i j k} \geq \tilde{b}_{j}, \forall j, \\
& \sum_{\forall i} \sum_{\forall j} x_{i j k} \leq \tilde{e}_{k}, \forall k, \\
& \mathrm{O} \leq x_{i j k} \leq l_{i j k} ;
\end{aligned}
$$

which is called the Uncertain Programming Model.

Since this uncertain mathematical model is difficult to handle, it can be solved utilizing any of the four ranking criteria given in section 2. In this paper, we have considered EVC and OVC to deal with the uncertain model. 


\subsection{Expected Value Model}

The core principle behind the EVM model is to optimize the problem by taking into account the expected values of all uncertain variables defined in the uncertain model (4.1). Mathematically, the EVM model for UMOCSTP can be expressed as follows:

\section{Model 4.1.1}

$E\left[Z_{t}\right]=E\left[\sum_{\forall i} \sum_{\forall j} \sum_{\forall k}\left(\xi_{i j k}^{t} x_{i j k}\right)\right], \forall t$

Subject to the constraints:

$E\left[\sum_{\forall j} \sum_{\forall k} x_{i j k}-\tilde{a}_{i}\right] \leq 0, \forall i$,

$E\left[\sum_{\forall i} \sum_{\forall k} x_{i j k}-\tilde{b}_{j}\right] \geq 0, \forall j$,

$E\left[\sum_{\forall i} \sum_{\forall j} x_{i j k}-\tilde{e}_{k}\right] \leq 0, \forall k$,

$0 \leq x_{i j k} \leq l_{i j k}$.

In this model, we will use notation $Z_{t E}$ to represent the objective functions $E\left[Z_{t}\right]$ formed by taking the expected values of $Z_{t}(x, \xi)$ throughout this paper.

\subsection{Optimistic Value Model}

To deal with uncertain models, an optimistic value model can also be developed by utilizing the optimistic value criterion defined for the uncertain variables. The OVM for the uncertain model (4.1) is given by:

\section{Model 4.2.1}

$Z_{\text {sup }}^{t}\left(\eta_{t}\right)=\left[\sum_{\forall i} \sum_{\forall j} \sum_{\forall k}\left(\xi_{i j k}^{t} x_{i j k}\right)\right]_{\text {sup }}\left(\eta_{t}\right), \forall t ;$

Subject to the constraints

$$
\begin{aligned}
& {\left[\sum_{\forall j} \sum_{\forall k} x_{i j k}-\tilde{a}_{i}\right]_{\text {sup }}\left(\alpha_{i}\right) \leq 0, \forall i,} \\
& {\left[\sum_{\forall i} \sum_{\forall k} x_{i j k}-\tilde{b}_{j}\right]_{\text {sup }}\left(\beta_{j}\right) \geq 0, \forall j,}
\end{aligned}
$$


$\left[\sum_{\forall i} \sum_{\forall j} x_{i j k}-\tilde{e}_{k}\right]_{\text {sup }}\left(\gamma_{k}\right) \leq 0, \forall k$

$0 \leq x_{i j k} \leq l_{i j k}$;

where $\eta_{t}, \alpha_{i}, \beta_{j}$, and $\gamma_{k}$ are the confidence levels assumed with some fixed values. In this model, we will use $Z_{t S}$ to represent the objective functions $Z_{\text {sup }}^{t}\left(\eta_{t}\right)$ formed by taking the optimistic values of $Z_{t}(x, \xi)$ throughout this paper.

\section{Deterministic Formulations}

To solve the proposed models involving uncertain variables in the constraints and objective functions, they are transformed into their deterministic forms using the expected values or optimistic values for computational ease.

\subsection{Expected Value Model}

Using the expected values of uncertain variables $\xi_{i j k}^{t}, \tilde{a}_{i}, \tilde{b}_{j}$, and $\tilde{e}_{k}$, the model (4.1.1) is equivalent to the model shown below:

\section{Model 5.1.1}

$\min Z_{t E}=\sum_{\forall i} \sum_{\forall j} \sum_{\forall k}\left(x_{i j k} \int_{0}^{1} \Phi_{\xi_{i j k}^{t}}^{-1}\left(\eta_{t}\right) d \eta_{t}\right), \forall t$

Subject to the constraints:

$$
\begin{aligned}
& \sum_{\forall j} \sum_{\forall k} x_{i j k}-\int_{0}^{1} \Phi_{\tilde{a}_{i}}^{-1}\left(\alpha_{i}\right) d \alpha_{i} \leq \mathrm{O}, \forall i, \\
& \int_{0}^{1} \Phi_{\widetilde{b}_{j}}^{-1}\left(\beta_{j}\right) d \beta_{j}-\sum_{\forall i} \sum_{\forall k} x_{i j k} \leq \mathrm{O}, \forall j, \\
& \sum_{\forall i} \sum_{\forall j} x_{i j k}-\int_{0}^{1} \Phi_{\widetilde{e}_{k}}^{-1}\left(\gamma_{k}\right) d \gamma_{k} \leq \mathrm{O}, \forall k, \\
& \mathrm{O} \leq x_{i j k} \leq l_{i j k}
\end{aligned}
$$

where $\eta_{t}, \alpha_{i}, \beta_{j}$, and $\gamma_{k}$ are the confidence levels with some fixed values.

\subsection{Optimistic Value Model}

The OVM model (4.2.1) is equivalent to the following model (5.2.1) obtained using the basic definitions and theorems defined in section 2 .

\section{Model 5.2.1}

$\min Z_{t S}=\sum_{\forall i} \sum_{\forall j} \sum_{\forall k} x_{i j k} \Phi_{\xi_{i j k}^{t}}^{-1}\left(1-\eta_{t}\right), \forall t$ 
Subject to the constraints:

$$
\begin{aligned}
& \sum_{\forall j} \sum_{\forall k} x_{i j k}-\Phi_{\tilde{a}_{i}}^{-1}\left(\alpha_{i}\right) \leq 0, \forall i, \\
& \Phi_{\tilde{b}_{j}}^{-1}\left(1-\beta_{j}\right)-\sum_{\forall i} \sum_{\forall k} x_{i j k} \leq 0, \forall j, \\
& \sum_{\forall i} \sum_{\forall j} x_{i j k}-\Phi_{\tilde{e}_{k}}^{-1}\left(\gamma_{k}\right) \leq 0, \forall k, \\
& 0 \leq x_{i j k} \leq l_{i j k}
\end{aligned}
$$

where $\eta_{t}, \alpha_{i}, \beta_{j}$ and $\gamma_{k}$ are the confidence levels with some fixed values.

\section{Solution Methodologies}

In this section, we discuss two main classical approaches for obtaining the compromise solution of the optimization problems with multiple objectives. These two approaches are minimizing distance method and fuzzy programming technique. We will utilize these methods to obtain the compromise solution for the crisp models of EVM and OVM.

\subsection{Minimizing Distance Method (MDM)}

This method is the most common approach to transform the multi-objective problems into a singleobjective problem and has been widely used in the literature. The single-objective model is formulated by minimizing the distance function given in equation (5) which minimizes the distance between the objective functions and their corresponding ideal values (Miettinen, 2008). This method utilizes $L_{2}$ norm to convert the crisp multi-objective EVM and OVM models into their corresponding single-objective model as given below:

$$
\min \sqrt{\sum_{t=1}^{S}\left(Z_{t}-Z_{t}^{o}\right)^{2}}
$$

Subject to the constraints of model (5.1.1) or (5.2.1).

Here, $Z_{t}$ represents the objective function for the EVM and OVM models whereas $Z_{t}^{o}=\min Z_{t}, t=1,2, \ldots S$, represents the ideal objective value of $Z_{t}$ in EVM and OVM models.

\subsection{Fuzzy Programming Technique (FPT)}

Zimmerman (1978) proposed the fuzzy programming technique to solve the linear programming problems with several objective functions. This technique converts the multi-objective problems to single-objective problem to obtain the solution. The steps of this fuzzy technique for the defined problem MOCSTP are sequentially given as follows:

Step 1. Solve each objective function of the deterministic models (5.1.1) and (5.2.1) as an individual single-objective problem w.r.t the given constraints by taking only one objective at a time and avoid rest of the other objectives. 
Step 2. Obtain the minimum $\left(L_{t}\right)$ and maximum $\left(U_{t}\right)$ values for all the $S$ objective functions individually to construct the linear membership function $\mu_{t}\left(Z_{t}\right)$ defined as:

$$
\mu_{t}\left(Z_{t}\right)= \begin{cases}1, & \text { if } Z_{t} \leq L_{t} \\ \frac{U_{t}-Z_{t}}{U_{t}-L_{t}}, & \text { if } L_{t} \leq Z_{t} \leq U_{t}, \\ 0, & \text { if } Z_{t} \geq U_{t}, \forall t\end{cases}
$$

Step 3. Now, the single-objective fuzzy model is formulated as

Maximize $\lambda$

Subject to the constraints:

$\mu_{t}\left(Z_{t}\right) \geq \lambda, t=1,2 \ldots S$,

and the constraints given in model 5.1.1 or 5.2.1

with $\lambda \geq 0$ and $\lambda=\min \left(\mu_{t}\left(Z_{t}\right)\right)$.

Step 4. Now, the converted single-objective problem obtained in step 3 is solved in LINGO 18.0 software to obtain the Pareto-optimal solution of the MOCSTP problem.

\section{Numerical Illustration}

Let us consider a TP in which we have three origins $m=3$, three destinations $n=3$, and two conveyances $K=2$ between origins and destinations. In this problem, we have considered all the parameters as independent zigzag uncertain variables. The problem aims at finding the total number of products to be shipped from sources to destinations with different conveyances such that the transportation cost and damage cost of items during the transportation are minimized. Here, the notations $\tilde{a}_{i}, \tilde{b}_{j}$, and $\tilde{e}_{k}$ are used to represent the supplier capacity, demand requirements and conveyance capacities respectively. The data for the considered MOCSTP problem is given in Tables 1 to 3 and the uncertain variables $\tilde{a}_{i}, \tilde{b}_{j}$, and $\tilde{e}_{k}$ are listed below:

$\tilde{a}_{1} \approx Z(10,12,13), \tilde{a}_{2} \approx Z(11,13,14), \tilde{a}_{3} \approx Z(12,14,16), \tilde{b}_{1} \approx Z(8,10,12), \tilde{b}_{2} \approx Z(9,10,11)$, $\tilde{b}_{3} \approx Z(10,11,12), \tilde{e}_{1} \approx Z(35,36,37), \tilde{e}_{2} \approx Z(40,41,42)$.

Table 1. The shipping costs for the two conveyances train and cargo ship.

\begin{tabular}{|c|c|c|c|c|c|c|c|}
\hline$\xi_{i j 1}^{1}$ & $\mathbf{1}$ & $\mathbf{2}$ & $\mathbf{3}$ & $\xi_{i j 2}^{1}$ & $\mathbf{1}$ & $\mathbf{2}$ & $\mathbf{3}$ \\
\hline 1 & $(2,4,6)$ & $(1,3,4)$ & $(3,4,5)$ & 1 & $(3,5,6)$ & $(2,3,4)$ & $(5,6,7)$ \\
\hline 2 & $(3,5,6)$ & $(4,5,6)$ & $(5,7,9)$ & 2 & $(7,8,9)$ & $(3,4,5)$ & $(5,6,8)$ \\
\hline 3 & $(1,2,3)$ & $(3,5,7)$ & $(4,5,6)$ & 3 & $(5,7,9)$ & $(4,6,8)$ & $(3,5,6)$ \\
\hline
\end{tabular}

Table 2. The damage costs for the two conveyances train and cargo ship.

\begin{tabular}{|c|c|c|c|c|c|c|c|}
\hline$\xi_{i j 1}^{2}$ & $\mathbf{1}$ & $\mathbf{2}$ & $\mathbf{3}$ & $\xi_{i j 2}^{2}$ & $\mathbf{1}$ & $\mathbf{2}$ & $\mathbf{3}$ \\
\hline 1 & $(4,6,8)$ & $(3,5,7)$ & $(2,3,4)$ & 1 & $(3,5,6)$ & $(6,7,8)$ & $(5,6,7)$ \\
\hline 2 & $(6,7,8)$ & $(5,6,7)$ & $(3,5,7)$ & 2 & $(2,4,6)$ & $(4,5,7)$ & $(2,4,5)$ \\
\hline 3 & $(6,7,9)$ & $(3,4,6)$ & $(5,7,8)$ & 3 & $(1,3,5)$ & $(3,5,6)$ & $(3,5,6)$ \\
\hline
\end{tabular}


Table 3. Fixed capacitated restriction on the route for both the conveyances.

\begin{tabular}{|c|c|c|c|}
\hline$i / j$ & $\mathbf{1}$ & $\mathbf{2}$ & $\mathbf{3}$ \\
\hline $\mathbf{1}$ & 6 & 7 & 8 \\
\hline $\mathbf{2}$ & 6 & 8 & 9 \\
\hline $\mathbf{3}$ & 10 & 12 & 13 \\
\hline
\end{tabular}

\section{Solution}

The uncertain model of the given problem defined above can be solved using the two deterministic models: EVM and OVM. These two models are formulated using the data values given in Tables 1 to 3 and their solution is obtained using the two methodologies stated in section 6 .

\subsection{Expected Value Model}

Formulating the model (7.1.1) with the objective function formed by taking the expected values of the data given as zigzag uncertain variables in Tables 1 to 3 .

\section{Model 7.1.1}

$\min Z_{1 E}=4 x_{111}+2.75 x_{121}+4 x_{131}+4.75 x_{211}+5 x_{221}+7 x_{231}+2 x_{311}+5 x_{321}+5 x_{331}$

$+4.75 x_{112}+3 x_{122}+6 x_{132}+8 x_{212}+4 x_{222}+6.25 x_{232}+7 x_{312}+6 x_{322}+4.75 x_{332}$;

$\min Z_{2 E}=6 x_{111}+5 x_{121}+3 x_{131}+7 x_{211}+6 x_{221}+5 x_{231}+7.25 x_{311}+4.25 x_{321}+6.75 x_{331}$

$+4.75 x_{112}+7 x_{122}+6 x_{132}+4 x_{212}+5.25 x_{222}+3.75 x_{232}+3 x_{312}+4.75 x_{322}+4.75 x_{332}$;

Subject to the constraints:

$\sum_{\forall j} \sum_{\forall k} x_{1 j k}-11.75 \leq 0 ; \sum_{\forall j} \sum_{\forall k} x_{2 j k}-12.75 \leq 0 ; \sum_{\forall j} \sum_{\forall k} x_{3 j k}-14 \leq 0 ;$

$10-\left(\sum_{\forall i} \sum_{\forall k} x_{i 1 k}\right) \leq 0 ; 10-\left(\sum_{\forall i} \sum_{\forall k} x_{i 2 k}\right) \leq 0 ; 11-\left(\sum_{\forall i} \sum_{\forall k} x_{i 3 k}\right) \leq 0$;

$\sum_{\forall i} \sum_{\forall j} x_{i j 1}-36 \leq 0 ; \sum_{\forall i} \sum_{\forall j} x_{i j 2}-41 \leq 0$;

$0 \leq x_{11 k} \leq 6 ; 0 \leq x_{12 k} \leq 7 ; 0 \leq x_{13 k} \leq 8$

$0 \leq x_{21 k} \leq 6 ; 0 \leq x_{22 k} \leq 8 ; 0 \leq x_{23 k} \leq 9$;

$0 \leq x_{31 k} \leq 10 ; 0 \leq x_{32 k} \leq 12 ; 0 \leq x_{33 k} \leq 13, k=1,2$.

We present here the results obtained for the EVM model (7.1.1) using the discussed two solution methodologies.

\section{(a) Minimizing Distance Method}

The results obtained using MDM are displayed below with ideal values taken as $Z_{1 E}^{o}=101.0625$ and $Z_{2 E}^{o}=112.8125$ :

$Z_{1 E}^{*}=125.6249, Z_{2 E}^{*}=141.7095, x_{121}=3.75, x_{131}=8, x_{311}=5.388, x_{321}=1, x_{222}=5.25, x_{312}=4.612, x_{332}=3$. 
(b) Fuzzy Programming Technique

For applying FPT with linear membership function, the $U_{t}$ and $L_{t}$ values are: $L_{1}=101.0625, L_{2}=112.8125, U_{1}=249.0625, U_{2}=258.3750$. Using these values, the compromise solution is obtained for the EVM model (7.1.1) which is given as:

$\lambda=0.8166, x_{121}=3.75, x_{131}=8, x_{311}=4.8706, x_{321}=1, x_{222}=5.25, x_{312}=5.1294, x_{332}=3$

with the objective values obtained as $Z_{1 E}^{*}=128.2096$ and $Z_{2 E}^{*}=139.5125$. Here, $\lambda$ is the minimum value of $\lambda_{1}$ and $\lambda_{2}$ i.e $\lambda=\min \left(\lambda_{1}, \lambda_{2}\right)$, where $\lambda_{1}=\mu_{1}\left(Z_{1 E}\right)$ and $\lambda_{2}=\mu_{2}\left(Z_{2 E}\right)$.

The fuzzy programming solution approach gives the minimum value $\lambda$ of these individual membership function values $\lambda_{1}, \lambda_{2}$, stating that each objective function possesses at least $\lambda$ degree of satisfaction level. As the membership value increases and approaches to 1 for the objective functions, the objective values are improved simultaneously and approach to the best value (optimal value) of the individual objective functions.

\subsection{Optimistic Value Model}

To formulate OVM, we need some predetermined confidence levels $\eta_{t}, \alpha_{i}, \beta_{j}, \gamma_{k} \in(0,1]$. Let us assume that all the confidence levels are equal to 0.9 .

\section{Model 7.2.1}

$$
\begin{aligned}
& \min Z_{1 S}=2.4 x_{111}+1.4 x_{121}+3.2 x_{131}+3.4 x_{211}+4.2 x_{221}+5.4 x_{231}+1.2 x_{311}+3.4 x_{321}+4.2 x_{331} \\
& +3.4 x_{112}+2.2 x_{122}+5.2 x_{132}+7.2 x_{212}+3.2 x_{222}+5.2 x_{232}+5.4 x_{312}+4.4 x_{322}+3.4 x_{332} \\
& \min Z_{2 S}=4.4 x_{111}+3.4 x_{121}+2.2 x_{131}+6.2 x_{211}+5.2 x_{221}+3.4 x_{231}+6.2 x_{311}+3.2 x_{321}+5.4 x_{331} \\
& +3.4 x_{112}+6.2 x_{122}+5.2 x_{132}+2.4 x_{212}+4.2 x_{222}+2.4 x_{232}+1.4 x_{312}+3.4 x_{322}+3.4 x_{332}
\end{aligned}
$$

Subject to the constraints:

$$
\begin{aligned}
& \sum_{\forall j} \sum_{\forall k} x_{1 j k}-12.8 \leq 0 ; \sum_{\forall j} \sum_{\forall k} x_{2 j k}-13.8 \leq 0 ; \sum_{\forall j} \sum_{\forall k} x_{3 j k}-15.6 \leq 0 ; \\
& 8.4-\left(\sum_{\forall i} \sum_{\forall k} x_{i 1 k}\right) \leq 0 ; 9.2-\left(\sum_{\forall i} \sum_{\forall k} x_{i 2 k}\right) \leq 0 ; 10.2-\left(\sum_{\forall i} \sum_{\forall k} x_{i 3 k}\right) \leq 0 ; \\
& \sum_{\forall i} \sum_{\forall j} x_{i j 1}-36.8 \leq 0 ; \sum_{\forall i} \sum_{\forall j} x_{i j 2}-41.8 \leq 0 ; \\
& 0 \leq x_{11 k} \leq 6 ; 0 \leq x_{12 k} \leq 7 ; 0 \leq x_{13 k} \leq 8 ; \\
& 0 \leq x_{21 k} \leq 6 ; 0 \leq x_{22 k} \leq 8 ; 0 \leq x_{23 k} \leq 9 ; \\
& 0 \leq x_{31 k} \leq 10 ; 0 \leq x_{32 k} \leq 12 ; 0 \leq x_{33 k} \leq 13, k=1,2 .
\end{aligned}
$$

The solution of this multi-objective OVM model (7.2.1) can be achieved with the two solution methodologies mentioned in section 6 . The results of both the methods are shown below.

\section{(a) Minimizing Distance Method}

The solution obtained for model (7.2.1) using MDM is: 
$x_{121}=7, x_{131}=5.8, x_{311}=3.1805, x_{321}=2.2, x_{312}=5.2195, x_{332}=4.4$ and the compromise values obtained for the objective functions are $Z_{1 S}^{*}=82.8018$ and $Z_{2 S}^{*}=85.5865$.

\section{(b) Fuzzy Programming Technique}

To apply the FPT with linear membership function, the $U_{t}$ and $L_{t}$ are taken as: $L_{1}=58.68, L_{2}=64.48, U_{1}=218.28, U_{2}=243.56$. The solution obtained for the OVM model (7.2.1) using linear membership function is given as:

$\lambda=0.8653, x_{121}=7, x_{131}=5.8, x_{311}=3.807, x_{321}=2.2, x_{312}=4.5930, x_{332}=4.4$

and the compromise values for the objective functions are $Z_{1 S}^{*}=80.1706$ and $Z_{2 S}^{*}=88.5936$. The fuzzy programming solution approach gives the minimum value $\lambda$ of these individual membership function values $\lambda_{1}, \lambda_{2}$, stating that each objective function possesses at least $\lambda$ degree of satisfaction level. As the membership value increases and approaches to 1 for the objective functions, the objective values are improved simultaneously and approach to the best value (optimal value) of the individual objective functions.

\section{Sensitivity Analysis of the Confidence Levels}

In this section, we investigate the sensitivity of the objective functions in the OVM model with respect to uncertain constraints. We performed the complementary test by varying the confidence levels $\alpha_{i}, \beta_{j}$, and $\gamma_{k}$ and taking $\eta_{t}=0.9$ in the objective function for all $i, j, k$, and $t$. The sensitivity analysis is done by changing the value of one confidence level between $[0.1,0.9]$ with a step increment of 0.1 and keeping the other confidence levels fixed as 0.9. For example, when we examine the sensitivity of $\alpha_{i}$ in the range [0.1,0.9], the values $\beta_{j}, \gamma_{k}$ are fixed as 0.9 . The results of the sensitivity analysis are only shown for FPT with linear membership function. The objective values obtained during the sensitivity analysis of OVM model are shown in Table 4 and the column "Variation in $\alpha_{i}$ " describes that only $\alpha_{i}$ is fluctuated between 0.1 to 0.9 and the rest of all the other confidence levels are kept fixed to 0.9. "CL" represents the variation of confidence level in $\alpha_{i}, \beta_{j}$, and $\gamma_{k}$. The graphical interpretation of the objective values w.r.t the confidence levels $\alpha_{i}, \beta_{j}$, and $\gamma_{k}$ is shown in Figure 1.

Figure 1 indicates that the objective function values are monotonically decreasing with respect to the confidence levels $\alpha_{i}, \beta_{j}$, and they remain constant with $\gamma_{k}$. As shown in the subplots of Figure 1 , the variation in the optimal values of the two objective functions with respect to the confidence level $\beta_{j}$ is extremely high and the variation in the optimal values with respect to $\alpha_{i}$ is moderately high whereas the objective values remain constant with the variation in confidence level $\gamma_{k}$. So, from Figure 1, we can clearly see that the best solution among all the solutions obtained during the sensitivity analysis is obtained when the values of $\alpha_{i}, \beta_{j}$, and $\gamma_{k}$ are assumed to be 0.9 and the worst solution is obtained when they are assumed as 0.1 . Therefore, we can say that the objective function values are improving with each step size increment in the value of confidence levels. 

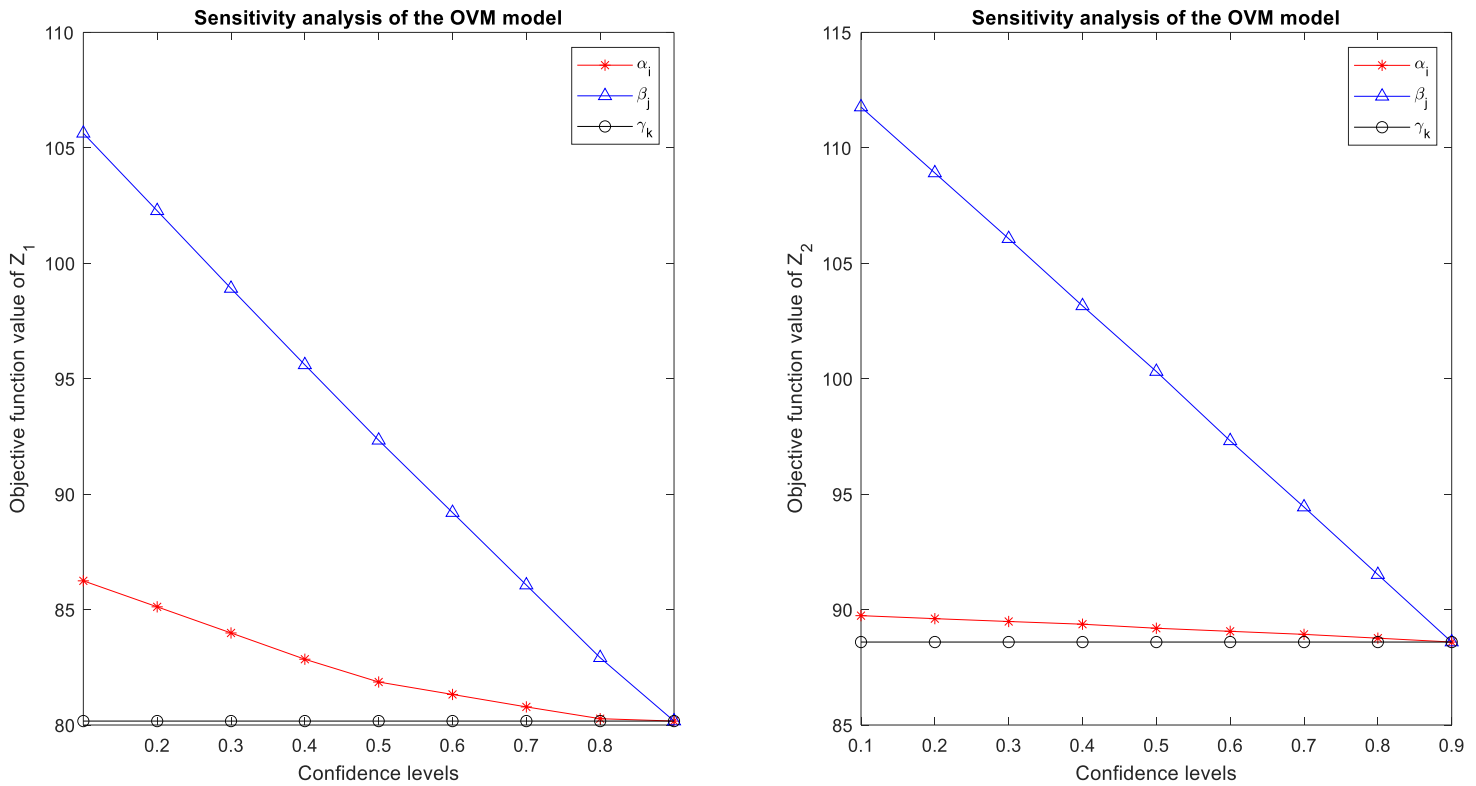

Figure 1. The sensitivity analysis for objectives of model (7.2.1) w.r.t $\alpha_{i}, \beta_{j}$ and $\gamma_{k}$ using FPT.

Table 4. Objective values obtained during the sensitivity analysis of model (7.2.1) with FPT.

\begin{tabular}{|c|c|c|c|c|}
\hline CL & Obj Func & Variation in $\alpha_{i}$ & Variation in $\beta_{j}$ & Variation in $\gamma_{k}$ \\
\hline \multirow{2}{*}{0.1} & $Z_{1 S}^{*}$ & 86.24508 & 105.6293 & 80.17058 \\
\hline & $Z_{2 S}^{*}$ & 89.73705 & 111.7665 & 88.59362 \\
\hline \multirow{2}{*}{0.2} & $Z_{1 S}^{*}$ & 85.11911 & 102.2730 & 80.17058 \\
\hline & $Z_{2 S}^{*}$ & 89.60673 & 108.9109 & 88.59362 \\
\hline \multirow{2}{*}{0.3} & $Z_{1 S}^{*}$ & 83.98692 & 98.90829 & 80.17058 \\
\hline & $Z_{2 S}^{*}$ & 89.48352 & 106.0648 & 88.59362 \\
\hline \multirow{2}{*}{0.4} & $Z_{1 S}^{*}$ & 82.84943 & 95.59973 & 80.17058 \\
\hline & $Z_{2 S}^{*}$ & 89.36637 & 103.1546 & 88.59362 \\
\hline \multirow{2}{*}{0.5} & $Z_{1 S}^{*}$ & 81.86268 & 92.33293 & 80.17058 \\
\hline & $Z_{2 S}^{*}$ & 89.19122 & 100.3109 & 88.59362 \\
\hline \multirow{2}{*}{0.6} & $Z_{1 S}^{*}$ & 81.32408 & 89.20053 & 80.17058 \\
\hline & $Z_{2 S}^{*}$ & 89.05820 & 97.37083 & 88.59362 \\
\hline \multirow{2}{*}{0.7} & $Z_{1 S}^{*}$ & 80.78462 & 86.0607 & 80.17058 \\
\hline & $Z_{2 S}^{*}$ & 88.92615 & 94.43910 & 88.59362 \\
\hline \multirow{2}{*}{0.8} & $Z_{1 S}^{*}$ & 80.27368 & 82.91401 & 80.17058 \\
\hline & $Z_{2 S}^{*}$ & 88.76150 & 91.51542 & 88.59362 \\
\hline \multirow{2}{*}{0.9} & $Z_{1 S}^{*}$ & 80.17058 & 80.17058 & 80.17058 \\
\hline & $Z_{2 S}^{*}$ & 88.59362 & 88.59362 & 88.59362 \\
\hline
\end{tabular}




\section{Results and Comparison}

The results for the uncertain MOCSTP determined using the EVM and OVM are shown in this section. Table 5 compares the results obtained for EVM and OVM models with minimizing distance method and fuzzy programming technique methodologies.

Table 5. Comparison of the results obtained with FPT and MDM.

\begin{tabular}{|l|c|c|c|}
\hline Model & Objective function & Fuzzy programming technique & Minimizing distance method \\
\hline \multirow{2}{*}{ EVM } & $Z_{1 E}^{*}$ & 128.2096 & 125.6249 \\
\cline { 2 - 4 } & $Z_{2 E}^{*}$ & 139.5125 & 141.7095 \\
\hline \multirow{2}{*}{ OVM } & $Z_{1 S}^{*}$ & 80.1706 & 82.8018 \\
\cline { 2 - 4 } & $Z_{2 S}^{*}$ & 88.5936 & 85.5865 \\
\hline
\end{tabular}

From the results obtained with the given solution methodologies, we can say that neither of the method is dominating the results of the other method because if one objective approaches towards its best value then the other objective value starts worsening. Also, the EVM model gives the solution in terms of expected values of the objective functions whereas OVM model gives the solution in terms of optimistic values of the objective functions. The results of the OVM obtained here are only for a single case of confidence level $\eta_{t}=0.9$ in the objective function, so numerous sets of solutions can be obtained by varying the $\eta_{t}$ in the range $(0,1]$.

\section{Conclusions}

In this study, a MOCSTP in an uncertain environment with zigzag uncertain variables is addressed. The uncertain MOCSTP model is first transformed into its deterministic EVM and OVM models using the expected and optimistic value criterion of uncertainty theory. Further, the multi-objective deterministic models were reduced to a single-objective model by employing MDM and FPT (with linear membership function). The solution technique for each method is illustrated using a numerical example, and the results obtained using both methods were compared. Finally, Paretooptimal solutions of both the methods were obtained using the Lingo 18.0 software. From the results, it is seen that none of the method is dominated by each other and act as an alternative approach for obtaining the compromise solution of uncertain MOCSTP, but if we use the exponential membership function in the fuzzy technique instead of linear membership function then a number of alternative solutions (due to shape parameters) can be obtained by this method unlike the minimizing distance method which gives only a single solution always. Also, it is noted that the EVM model will always lead to a single solution whereas the OVM model will always provide numerous solutions to the decision-maker because of the confidence levels involved in the OVM model. So, the OVM model can give the decision-maker with a number of alternative solutions by varying the confidence levels than the EVM model.

\section{Scope for Future Work}

This paper focuses on MOCSTP in the uncertain environment with zigzag uncertain variables and the results have been obtained using the fuzzy programming technique with linear membership function. In future, the same MOCSTP problem can be solved by employing different membership functions (like exponential or hyperbolic) in the fuzzy programming technique. In addition, other uncertain environments such as uncertain random environments or uncertain intervals can be 
considered and the future work can also be extended by studying the MOCSTPs under twofold uncertainty.

\section{Conflict of Interest}

Both the authors declare that they have no conflict of interest.

\section{Acknowledgements}

The authors are grateful to all the anonymous referees for their valuable comments and suggestions which helped in improving the quality of the paper. The first author would also like to extend her gratitude to the Council of Scientific \& Industrial Research, File No.09/1007(0003)/2017-EMR-I, New Delhi, India for providing financial support to this research work.

\section{References}

Acharya, D. (2016). Generalized solid capacitated transportation problem. South Asian Journal of Mathematics, 6(1), 24-30.

Ahmadi, K. (2018). On solving capacitated transportation problem. Journal of Applied Research on Industrial Engineering, 5(2), 131-145.

Bhargava, A.K., Singh, S.R., \& Bansal, D. (2014). Multi-objective fuzzy chance constrained fuzzy goal programming for capacitated transportation problem. International Journal of Computer Applications, 107(3), 18-23.

Chen, B., Liu, Y., \& Zhou, T. (2019). An entropy based solid transportation problem in uncertain environment. Journal of Ambient Intelligence and Humanized Computing, 10(1), 357-363.

Cui, Q., \& Sheng, Y. (2013). Uncertain programming model for solid transportation problem. International Information Institute (Tokyo). Information, 16(2), 1207-1213.

Ebrahimnejad, A. (2015). An improved approach for solving fuzzy transportation problem with triangular fuzzy numbers. Journal of Intelligent \& Fuzzy Systems, 29(2), 963-974.

Gao, Y., \& Kar, S. (2017). Uncertain solid transportation problem with product blending. International Journal of Fuzzy Systems, 19(6), 1916-1926.

Giri, P.K., Maiti, M.K., \& Maiti, M. (2014). Fuzzy stochastic solid transportation problem using fuzzy goal programming approach. Computers \& Industrial Engineering, 72, 160-168.

Gupta, N., \& Bari, A. (2014). Fuzzy multi-objective capacitated transportation problem with mixed constraints. Journal of Statistics Applications and Probability, 3(2), 1-9.

Gupta, S., Ali, I., \& Ahmed, A. (2018). Multi-choice multi-objective capacitated transportation problem- A case study of uncertain demand and supply. Journal of Statistics and Management Systems, 21(3), 467491.

Gupta, S., Ali, I., \& Ahmed, A. (2020). An extended multi-objective capacitated transportation problem with mixed constraints in fuzzy environment. International Journal of Operational Research, 37(3), 345-376.

Hassin, R., \& Zemel, E. (1988). Probabilistic analysis of the capacitated transportation problem. Mathematics of Operations Research, 13(1), 80-89.

Hitchcock, F.L. (1941). The distribution of a product from several sources to numerous localities. Journal of Mathematics and Physics, 20(1-4), 224-230.

Kakran, V.Y., \& Dhodiya, J.M. (2021). Uncertain multi-objective transportation problems and their solution. In Patnaik, S., Tajeddini, K., Jain, V. (eds) Computational Management. Springer, Cham, pp. 359-380. 
Kolmogorov, A.N., \& Bharucha-Reid, A.T. (2018). Foundations of the theory of probability: second english edition. Courier Dover Publications, Mineola, New York.

Liu, B. (2007). Uncertainty theory. In Baoding, L. (ed) Uncertainty theory. Springer, Berlin, Heidelberg. pp. 205-234.

Liu, B. (2010). Uncertainty theory. In Baoding, L. (ed) Uncertainty theory. Springer, Berlin, Heidelberg. pp. $1-79$.

Liu, B., \& Liu, B. (2009). Theory and practice of uncertain programming. Springer, Berlin, Heidelberg.

Lohgaonkar, M., \& Bajaj, V. (2010). Fuzzy approach to solve multi-objective capacitated transportation problem. International Journal of Bioinformatics Research, 2(1), 10-14.

Miettinen, K. (2008). Introduction to multiobjective optimization: noninteractive approaches. In Branke, J., Deb, K., Miettinen, K., Slowinski, R. (eds) Multiobjective optimization. Springer, Berlin, Heidelberg. pp. 1-26.

Moore, R.E., \& Yang, C.T. (1996). Interval analysis (Vol. 2). Englewood Cliffs, NJ: Prentice-Hall.

Mou, D., Zhao, W., \& Chang, X. (2013). A transportation problem with uncertain truck times and unit costs. Industrial Engineering and Management Systems, 12(1), 30-35.

Panda, A., \& Das, C.B. (2014). Capacitated transportation problem under vehicles. LAP LAMBERT Academic Publisher, Deutschland/Germany.

Sadia, S., Gupta, N., \& Ali, Q.M. (2016). Multiobjective capacitated fractional transportation problem with mixed constraints. Mathematical Sciences Letters, 5(3), 235-242.

Schell, E. (1955). Distributuin of s product by several properties. In: Direstorate of Management Analysis, Proc. of the second Symposium in Linear Programming (Vol. 2, pp. 615-642). DCS/Comptroller HQUSAF. Washington.

Sharma, S., \& Arora, S. (2021). Bi-objective capacitated transportation problem with bounds over distributions and requirement capacities. International Journal of Applied and Computational Mathematics, 7(3), 1-14.

Wagner, H.M. (1959). On a class of capacitated transportation problems. Management Science, 5(3), 304318.

Zadeh, L.A. (1996). Fuzzy sets. In: Klir, G.J., Yuan, B. (eds) Fuzzy sets, fuzzy logic, and fuzzy systems: selected papers by Lotfi A Zadeh. World Scientific, Singapore, pp. 394-432.

Zhao, G., \& Pan, D. (2020). A transportation planning problem with transfer costs in uncertain environment. Soft Computing, 24(4), 2647-2653.

Zimmermann, H.J. (1978). Fuzzy programming and linear programming with several objective functions. Fuzzy Sets and Systems, 1(1), 45-55.

Original content of this work is copyright @International Journal of Mathematical, Engineering and Management Sciences. Uses under the Creative Commons Attribution 4.0 International (CC BY 4.0) license at https://creativecommons.org/licenses/by/4.0/ 